\title{
Long-Range Models of Modified Gravity and Their Agreement with Solar System and Double Pulsar Data
}

\author{
Lorenzo lorio* \\ INFN-Sezione di Pisa \\ E-mail: lorenzo.iorio@libero.it
}

\begin{abstract}
Many long-range modifications of the Newtonian/Einsteinian standard laws of gravity have been proposed in the recent past to explain various celestial phenomena occurring at different scales ranging from solar system to the entire universe. The most famous ones are the so-called Pioneer anomaly, i.e. a still unexplained acceleration detected in the telemetry of the Pioneer 10/11 spacecraft after they passed the $20 \mathrm{AU}$ threshold in the solar system, the non-Keplerian profiles of the velocity rotation curves of several galaxies and the cosmic acceleration. We use the latest observational determinations of the planetary motions in the solar system and in the double pulsar system to put constraints on such models independently of the phenomena for which they were originally proposed. We also deal with the recently detected anomalous perihelion precession of Saturn and discuss the possibility that it can be explained by some of the aforementioned models of modified gravity.
\end{abstract}

5th International School on Field Theory and Gravitation, April 20 - 242009

Cuiabá city, Brazil

${ }^{*}$ Speaker. 


\section{Motivations for Long-Range Modifications of Gravity}

Historically, the first attempt to modify the laws of gravity commonly accepted at that time was due to Laplace [1] who, in 1805, tried to add a velocity-dependent term to the standard inversesquare law of Newton to account for the finite velocity of propagation of gravity. But this work did not find echo practically until the surroundings of 1880 , when a series of works to estimate the gravitational finite propagation speed began. Such attempts to find deviations from the Newtonian inverse-square law of gravitation were performed to explain the anomalous secular precession of Mercury's perihelion, discovered by Le Verrier [2], without invoking undetected (baryonic) matter like the hypothesized planet Vulcan: for example, Hall [3] noted that he could account for Mercury's precession if the law of gravity, instead of falling off as $1 / r^{2}$, actually falls of as $1 / r^{k}$ with $k=2.00000016$. However, such an idea was not found to be very appealing, since it conflicts with basic conservation laws, e.g., Gauss Law, unless one also postulates a correspondingly modified metric for space. Other historical attempts to modify Newton's law of gravitation to account for the Mercury's perihelion behavior yielded velocity-dependent additional terms: for a review of them see Ref. [4] and references therein. Such attempts practically ceased after the successful explanation of the perihelion rate of Mercury by Einstein [5] in terms of his tensorial general theory of relativity: an exception is represented by Manev [6] who, with a $1 / r^{2}$ correction to the Newtonian potential, was able to reproduce the anomalous apsidal precession of Mercury.

Moving to more recent times, in the modern framework of the challenge of unifying gravity with the other three fundamental interactions of Nature, it was realized that possible new phenomena could show up just as deviations from the Newtonian inverse-square law of gravitation [7, 8]. In general, they would occur at submillimeter length scales. Concerning general relativity, traditionally, corrections to it, in the form of modifications of the Einstein-Hilbert action in order to include higher-order curvature invariants with respect to the Ricci scalar, were considered to be important only at scales close to the Planck one and, consequently, in the early universe or near black hole singularities ${ }^{1}$; see, e.g., Ref [9] and references therein. It was not expected that such corrections could affect the gravitational phenomenology at low energies, and consequently at larger, macroscopic scales.

Thus, why considering modifications of the standard laws of gravity at large, astronomical, astrophysical and cosmological as well, as done in recent years? To try to accommodate some recently observed phenomena, occurring at very different scales ranging from solar system to cosmological distances, which, at present, have not yet found fully satisfactorily mundane explanations in terms of conventional physics, gravitational or not $[10,11,12]$. Below we list just some of such anomalous effects.

- The flyby anomaly. It consists of unexplained changes of the asymptotic outgoing velocities of some spacecraft (Galileo, NEAR, Cassini, and MESSENGER) that occurred at their closest approaches with the Earth [13,14]. Is it due to conventional non-conservative effects, or are modifications of the laws of gravity responsible of it?

\footnotetext{
${ }^{1}$ It was shown that in such ways the non-renormalizability of general relativity became more tractable in the context of quantum field theory.
} 
- The anomalous perihelion precession of Saturn [15] detected by processing almost one century of planetary observations with the inclusion of the latest radiometric data of the Cassini spacecraft [16]: is it a data processing artifact or a genuine physical effect?

- The Pioneer anomaly. It is an unexplained acceleration $A_{\text {Pio }}=(8.74 \pm 1.33) \times 10^{-10} \mathrm{~m} \mathrm{~s}^{-2}$ approximately directed towards the Sun affecting the Pioneer 10/11 spacecraft after they passed the $20 \mathrm{AU}$ threshold in the solar system [17, 18]. Is it induced by some mundane non-conservative effects, or is it a sign of modifications of the laws of gravity?

- The dark matter issue. In many astrophysical systems like, e.g., spiral galaxies and clusters of galaxies a discrepancy between the observed kinematics of their exterior parts and the predicted one on the basis of the Newtonian dynamics and the matter detected from the emitted electromagnetic radiation (visible stars and gas clouds) was present since the pioneering studies by Zwicky [19] on the Coma cluster, and by Kahn and Woltjer [20], Bosma [21], and Rubin and coworkers [22, 23] on individual galaxies. More precisely, such an effect shows up in the galactic velocity rotation curves [24] whose typical pattern after a few kpc from the center differs from the Keplerian $1 / \sqrt{r}$ fall-off expected from the usual dynamics applied to the electromagnetically-observed matter. Does the cause of such a phenomenology reside in the action of still undetected (non-baryonic) dark matter whose dynamics is governed by the usual Newtonian laws of gravitation, or have they to be modified?

- The dark energy issue. In recent years, an increasing amount of observational evidence has accumulated pointing toward the fact that the universe has entered a phase of accelerating expansion. Some of such observations are of direct, geometrical nature: standard candles like the supernovæ SnIa [25, 26], gamma ray bursts [27] and standard rulers like the CMB sound horizon $[28,29]$. Other ones are of dynamical nature like the rate of growth of cosmological perturbations [30] probed by the redshift distortion factor or by weak lensing [31]. All these observations are converging towards a confirmation of the accelerating expansion of the universe, assumed homogeneous. They are successfully fitted by the simplest cosmological model predicting accelerating cosmic expansion: its ingredients are the the assumptions of flatness, validity of general relativity, the presence of a cosmological constant $\Lambda$, identified with an unknown and still directly undetected form of energy (named dark energy for these reasons), and Cold Dark Matter $(\Lambda \mathrm{CDM})$. However, for some puzzles of the $\Lambda \mathrm{CDM}$ cosmology, see Ref. [32]. Contrary to the $\Lambda \mathrm{CDM}$ paradigm, is it possible to accommodate the aforementioned observations by invoking modifications of the standard laws of gravity?

Some of the models of modified gravity that have been proposed to address the aforementioned phenomenology are listed below.

- Dvali-Gabadadze-Porrati (DGP) braneworld model [33]. In it our universe is a (3+1) spacetime brane embedded in a five-dimensional Minkowskian bulk. All the particles and fields of our experience are constrained to remain on the brane apart from gravity which is free to explore the empty bulk. Beyond a certain threshold $r_{0}$, which is a free-parameter of the theory and is fixed by observations to about $5 \mathrm{Gpc}$, gravity experiences strong modifications with respect to the usual four-dimensional Newton-Einstein picture: they allow to explain 
the observed acceleration of the expansion of the Universe without resorting to the concept of dark energy.

- MOdified Gravity (MOG) by Moffat [34]. It is a fully covariant theory of gravity which is based on the existence of a massive vector field coupled universally to matter. MOG yields a Yukawa-like modification of gravity with three constants which, in the most general case, are running; they are present in the theory's action as scalar fields which represent the gravitational constant, the vector field coupling constant, and the vector field mass. It has used to successfully describe various observational phenomena on astrophysical and cosmological scales without resorting to dark matter [35].

- MOdified Newtonian Dynamcs (MOND) [36, 37, 38]. It is a non-linear theory of gravity which predicts departures from the standard Newtonian inverse-square law at a characteristic acceleration scale [39] $A_{0}=1.27 \times 10^{-10} \mathrm{~m} \mathrm{~s}^{-2}$ below which the gravitational acceleration gets $\mathrm{a} \approx 1 / r$ behavior. MOND was proposed to explain certain features of the motion of ordinary electromagnetically detectable matter in galaxies and of galaxies in galactic clusters without resorting to exotic forms of still undetected dark matter.

- $f(R)$ models [40]. These theories come about by a straightforward generalization of the Lagrangian in the Einstein-Hilbert action in which the Ricci scalar $R$ is replaced by a general analytical function $f(R)$ of $R$. They have mainly been used in cosmological and astrophysics scenarios [41].

- Curvature Invariants models. They encompass inverse powers [42, 43] and logarithm [44] of some curvature invariants in the Einstein-Hilbert action and have been used for tackling the dark energy-dark matter problems.

- Yukawa-like models. There are many theoretical frameworks yielding such a modification of the Newtonian inverse-square law [45, 46, 12]. Models encompassing Yukawa-type extraaccelerations have been used for a variety of applications ranging from solar system effects like the Pioneer anomaly [34] to astrophysical and cosmological scenarios [47, 48, 49, 50, $51,52]$.

- Hooke-like models. With such a definition we mean models of gravity introducing an additional term proportional to the distance $r$. An important case is given by the Schwarzschild-de Sitter spacetime [53] which yields a correction to the Newtonian inverse-square law proportional to $\Lambda r$ [54], where $\Lambda$ is an uniform cosmological constant. Another example is given by the extra-acceleration proposed by Jaekel and Reynaud [55] to explain the Pioneer anomaly.

- Pioneer-like models. The simplest one consists of postulating a constant and uniform acceleration radially directed towards the Sun and having the same magnitude of $A_{\text {Pio }}$ existing in the outer regions of the solar system at heliocentric distances $r \geq 20 \mathrm{AU}$. Other forms have been postulated for it, both distance-dependent $[55,56]$ and velocity-dependent $[57,58,60]$.

Other models of modified gravity that we will not consider here are the Einstein-Aether theory [61], Tensor-Vector-Scalar (TeVeS) [62] and braneworld gravity [63]. 
Table 1: Corrections $\Delta \dot{\varpi}$, in $10^{-4} \operatorname{arcsec~cy}^{-1}$, to the standard Newton/Einstein perihelion precessions of the inner planets estimated by E.V. Pitjeva with the EPM2004 [66] (Mercury, Earth, Mars) and EPM2006 [67] (Venus) ephemerides. The result for Venus has been obtained by recently processing radiometric data from Magellan spacecraft (E.V. Pitjeva, personal communication, 2008). The errors in square brackets are the formal ones: the other ones have been re-scaled by Pitjeva to get realistic estimates.

\begin{tabular}{cccc}
\hline Mercury & Venus & Earth & Mars \\
\hline$-36 \pm 50[42]$ & $-4 \pm 5[1]$ & $-2 \pm 4[1]$ & $1 \pm 5[1]$ \\
\hline
\end{tabular}

The only motivations for the aforementioned models are just the phenomena themselves for which they have been introduced. Thus, such models must be put on the test in different scenarios by devising independent observational checks. In particular, they must not exhibit discrepancies with the well-tested standard laws of gravity in local astronomical systems like, e.g., our solar system and the double pulsar PSR J0737-3039 [64, 65].

\section{Our Method for Testing Modified Models of Gravity in Astronomical Scenarios}

In general, a given Long Range Modified Model of Gravity (LRMMG) yields predictions $\mathscr{P}$ for certain observable effects $\mathscr{O}$ of the form

$$
\mathscr{P}=K g,
$$

where $K \rightarrow 0$ implies no modifications of gravity, and $g$ is a function of the geometrical configuration of the system adopted characteristic of the LRMMG considered; the fact that the LRMMG parameter $K$ enters as a multiplicative factor in the predicted effects $\mathscr{P}$ will be very important for us, as we will see below. For example, for the solar system's planetary longitudes of the perihelia $\varpi$, it turns out

$$
g=g(a, e)
$$

with $a$ semimajor axis and $e$ eccentricity of the planetary orbit considered. Corrections $\Delta \dot{\varpi}$ to the usual Newtonian/Einsteinian perihelion precessions have been recently estimated for several planets of the solar system by E.V. Pitjeva $[66,67,16]$ by fitting $100 \mathrm{yr}$ of observations of several kinds with the force models of various versions of the EPM ephemerides (EPM2004 [68] EPM2006 [67], EPM2008 [16]). Since they do not include any LRMMGs, the corrections $\Delta \dot{\varpi}$, by construction, account for them, so that they will be our $\mathscr{O}$ : they are listed in Table 1 . By directly comparing $\Delta \dot{\Phi}$ to the predicted anomalous perihelion precession $\mathscr{P}=K g(a, e)$ for each planet separately allows to put upper bounds on $|K|$ since $\Delta \dot{\Phi}$ are compatible with zero, according to Table 1 . This approach is good for test the hypothesis $K=0$. The hypothesis $K \neq 0$ can be tested by taking the ratios

$$
\Pi_{\mathrm{AB}} \equiv \frac{\Delta \dot{\varpi}_{\mathrm{A}}}{\Delta \dot{\varpi}_{\mathrm{B}}}
$$

of $\Delta \dot{\varpi}$ for different pairs of planets $\mathrm{A}$ and $\mathrm{B}$, and comparing them to the predicted ratios

$$
\xi_{\mathrm{AB}} \equiv \frac{\mathscr{P}_{\mathrm{A}}}{\mathscr{P}_{\mathrm{B}}}=\frac{g\left(a_{\mathrm{A}}, e_{\mathrm{A}}\right)}{g\left(a_{\mathrm{B}}, e_{\mathrm{B}}\right)}:
$$


Table 2: Ratios $\Pi_{\mathrm{AB}}$ of the estimated corrections of the perihelia $\Delta \dot{\varpi}$ for the pairs of planets $\mathrm{A} B$. The uncertainties $\delta \Pi_{\mathrm{AB}}$ have been evaluated by using the realistic errors for the individual corrections of Table 1 .

\begin{tabular}{llrr}
\hline A & B & $\Pi_{\mathrm{AB}}$ & $\delta \Pi_{\mathrm{AB}}$ \\
\hline Mercury & Venus & 9 & 24 \\
Venus & Mercury & 0.1 & 0.3 \\
Mercury & Earth & 18 & 61 \\
Earth & Mercury & 0.06 & 0.19 \\
Mercury & Mars & -36 & 230 \\
Mars & Mercury & -0.03 & 0.18 \\
Venus & Earth & 2 & 6.5 \\
Earth & Venus & 0.5 & 1.6 \\
Venus & Mars & -4 & 25 \\
Mars & Venus & -0.2 & 1.6 \\
Earth & Mars & -2 & 14 \\
Mars & Earth & -0.5 & 3.5 \\
\hline
\end{tabular}

$\xi_{\mathrm{AB}}$, by construction, does not explicitly depend on $K$, but it still retains a functional dependence on $a$ and $e$ typical of the LRMMG considered. If

$$
\Pi_{\mathrm{AB}} \neq \xi_{\mathrm{AB}}
$$

within the observational errors, i.e. if

$$
\Psi_{\mathrm{AB}} \equiv\left|\Pi_{\mathrm{AB}}-\xi_{\mathrm{AB}}\right| \neq 0
$$

the LRMMG considered is severely challenged. Of course, the uncertainty in the ratios $\Pi_{A B}$ has to be evaluated in a realistic and conservative way to reduce the risk of unsound conclusions; see the discussion in Section 4. We quote the ratios $\Pi_{\mathrm{AB}}$ for all the pairs of the inner planets in Table 2.

An analogous approach can be followed with the double pulsar PSR J0737-3039. Now, one observable is $\Delta \dot{\omega}$, i.e. the difference between the phenomenologically determined periastron precession $\dot{\omega}_{\text {meas }}$ and the computed rate $\dot{\omega}_{1 \mathrm{PN}}$ for the general relativistic $1 \mathrm{PN}$ rate (it is analogous to the well-known Mercury's perihelion precession by Einstein). Another observable is $\Delta P$, i.e. the difference between the phenomenologically determined orbital period $P_{\mathrm{b}}$ and the computed Keplerian one $P^{\mathrm{Kep}}$. Thus, the observation-based ratio

$$
\mathscr{R} \equiv \frac{\Delta \dot{\omega}}{\Delta P}
$$

can be compared with the predictions $\mathscr{P}$ for the same ratio by various LRMMGs; again, if they are equal within the errors, LRRMMG passes the test, otherwise it is challenged. 


\section{Results from Local Systems}

\subsection{Dvali-Gabadadze-Porrati (DGP) Braneworld Model}

The DGP braneworld model [33], put forth to explain the cosmic acceleration without resorting to dark energy, predicts an anomalous perihelion precession independent of the semimajor axis $[69,70,71]$

$$
\dot{\Phi}_{\mathrm{DGP}}=\mp \frac{3 c}{8 r_{0}}=\mp 0.0005 \operatorname{arcsec}^{-1}, r_{0} \approx 5 \mathrm{Gpc},
$$

where the $\mp$ sign are related to the two different cosmological branches of the model. Thus,

$$
\xi_{\mathrm{AB}}=1
$$

for it. By linearly adding the absolute values of the uncertainties in $\Delta \dot{\Phi}$, it turns out for the pairs $\mathrm{A}=$ Mars, $\mathrm{B}=$ Mercury and $\mathrm{A}=$ Earth, B=Mercury [72]

$$
\begin{aligned}
& \Psi_{\text {Mars Mercury }}=1.0 \pm 0.2, \text { ruled out at } 5 \sigma \text { level } \\
& \Psi_{\text {Earth Mercury }}=0.9 \pm 0.2, \text { ruled out at } 4.5 \sigma \text { level }
\end{aligned}
$$

In the case of the double pulsar [73], the predicted ratio $\mathscr{R}_{\text {DGP }}$ between $\dot{\omega}_{\text {DGP }}$ and $P_{\text {DGP }}$ is

$$
\mathscr{R}_{\text {DGP }}=(1.4 \pm 0.1) \times 10^{-7} \mathrm{~s}^{-2} .
$$

Instead, the observed ratio $\mathscr{R}_{\mathrm{obs}}$ between $\Delta \dot{\omega} \equiv \dot{\omega}_{\text {meas }}-\dot{\omega}_{1 \mathrm{PN}}$ and $\Delta P=P_{\mathrm{b}}-P^{\mathrm{Kep}}$ is

$$
\mathscr{R}_{\text {meas }}=(0.3 \pm 4) \times 10^{-11} \mathrm{~s}^{-2} .
$$

They are not compatible at $14 \sigma$ level.

\subsection{MOdified Gravity (MOG)}

MOG [34] predicts a Yukawa-type extra-acceleration [74, 75]

$$
A_{\mathrm{MOG}}=-\frac{G M}{r^{2}} \alpha[1-(1+\mu r) \exp (-\mu r)],
$$

where $\alpha$ and $\mu$ have been fitted to a set of astrophysical galactic data [75] in the framework of the searches for an explanation of the flat rotation curves of galaxies without resorting to dark matter. For a Sun-planet system we have a perihelion extra-rate $(1 / \mu=33,000 \mathrm{AU})$ [76]

$$
\dot{\Phi}_{\mathrm{MOG}} \approx-\alpha \mu^{2} \sqrt{G M a\left(1-e^{2}\right)} .
$$

The inner planets yield [76]

$$
\begin{aligned}
& \Psi_{\text {Venus Mercury }}=1.3 \pm 0.3, \text { ruled out at } 4 \sigma \text { level } \\
& \Psi_{\text {Earth Mercury }}=1.6 \pm 0.2, \text { ruled out at } 8 \sigma \text { level } \\
& \Psi_{\text {Mars Mercury }}=2 \pm 0.2, \text { ruled out at } 10 \sigma \text { level }
\end{aligned}
$$




\subsection{MOdified Newtonian Dynamics (MOND)}

MOND [36, 37, 38] predicts acceleration-dependent modifications of the Newtonian inversesquare law to explain the almost flat galactic rotation curves

$$
A=\frac{A_{\text {Newton }}}{\mu(x)}, x \equiv \frac{A}{A_{0}},
$$

where galaxies data fitting yields [39] $A_{0}=1.2 \times 10^{-10} \mathrm{~m} \mathrm{~s}^{-2}$ and $\mu(x)$ is an interpolating function whose most widely used forms are

$$
\mu=\frac{x}{\sqrt{1+x^{2}}}[77], \mu=\frac{x}{1+x}[78]
$$

with

$$
\mu \rightarrow 1, x \gg 1
$$

and

$$
\mu \rightarrow x, x \ll 1 .
$$

solar system observations are, strictly speaking, tests of the form of the interpolating function in the large acceleration limit $(x \gg 1)$.

The anomalous perihelion precession predicted by MOND is, in the large acceleration limit $(x \gg 1),[36,79,80]$

$$
\dot{\varpi}_{\mathrm{MOND}}=-k_{0} n\left(\frac{a}{r_{\mathrm{MOND}}}\right)^{2 h} h, n=\sqrt{\frac{G M}{a^{3}}}, r_{\mathrm{MOND}}=\sqrt{\frac{G M}{A_{0}}} ;
$$

$k_{0}=1, h=1$ correspond to

$$
\mu=\frac{x}{1+x}
$$

$k_{0}=1 / 2, h=2$ correspond to

$$
\mu=\frac{x}{\sqrt{1+x^{2}}} .
$$

It turns out [81] that the ratios $\Pi_{\mathrm{AB}}$ of the perihelia, independent by construction of $k_{0}$, for the pairs $\mathrm{A}=$ Mars, $\mathrm{B}=$ Mercury and $\mathrm{A}=$ Earth, $\mathrm{B}=$ Mercury rule out the MOND perihelion precessions for $1 \leq h \leq 2$ at several sigma level.

\subsection{Extended $f(R)$ theories}

Extended theories of gravity with [82]

$$
f(R)=f_{0} R^{k}
$$

where $R$ is the Ricci scalar, yield power-law corrections to the Newton's law

$$
A_{f(R)}=\frac{(\beta-1) G M}{r_{c}^{\beta}} r^{\beta-2}
$$


which, for $\beta=0.817$, obtained by fitting the SNeIa Hubble diagram, yields good results in fitting some galactic rotation curves; $\beta$ is related to the exponent $k$ of the Ricci scalar $R$. The induced perihelion precession is [83]

$$
\dot{\omega}_{f(R)}=\frac{(\beta-1) \sqrt{G M}}{2 \pi r_{c}^{\beta}} a^{\beta-3 / 2} G(e, \beta),
$$

where $G(e, \beta)$ is a function of the eccentricity and $\beta$. The resulting $\Psi_{\mathrm{AB}}$ are not compatible with zero for many pairs A and B of inner and outer planets at several sigma level [83].

\subsection{Inverse powers and logarithm of some curvature invariants models}

Inverse powers of curvature invariants in the action lead to [42, 43]

$$
A_{\text {inv. pow. }}=-\frac{\alpha G M(6 k+3)}{r_{c}^{6 k+4}} r^{6 k+2}
$$

For $k=1$ and the Sun, $r_{c}=10 \mathrm{pc}$, so that

$$
\dot{\Phi}_{k=1} \approx-\frac{45 \alpha}{r_{c}^{10}} \sqrt{G M a^{17}\left(1-e^{2}\right)}
$$

Thus, the inner planets yield [72]

$$
\begin{gathered}
\Psi_{\text {Mars Mercury }}=10^{5} \pm 0.1, \text { ruled out at } 10^{6} \sigma \text { level } \\
\Psi_{\text {Mars Earth }}=38 \pm 3.5, \text { ruled out at } 11 \sigma \text { level } \\
\Psi_{\text {Earth Mercury }}=10^{3} \pm 0.2, \text { ruled out at } 10^{4} \sigma \text { level }
\end{gathered}
$$

Logarithm of some curvature invariants in the action, able to modify gravity at MOND scales so to jointly treat Dark Matter and Dark Energy, induces [44]

$$
A_{\mathrm{Log}} \propto \frac{r^{2}}{r_{c}^{4}}, r_{c}=0.04 \mathrm{pc}
$$

so that

$$
\dot{\varpi}_{\log } \propto \alpha \frac{\sqrt{G M a^{5}\left(1-e^{2}\right)}}{r_{c}^{4}}
$$

The pairs A=Mars, B=Mercury and A=Earth, B=Mercury yield [72]

$$
\begin{aligned}
& \Psi_{\text {Mars Mercury }}=30.7 \pm 0.1, \text { ruled out at } 31 \sigma \text { level } \\
& \Psi_{\text {Earth Mercury }}=10.6 \pm 0.2, \text { ruled out at } 53 \sigma \text { level }
\end{aligned}
$$




\subsection{Yukawa-like theories}

Many theoretical frameworks $[45,46,12]$ yield a Yukawa-type acceleration

$$
A_{\text {Yukawa }}=-\frac{G M \alpha}{r^{2}}\left(1+\frac{r}{\lambda}\right) \exp \left(-\frac{r}{\lambda}\right) \text {. }
$$

For $\lambda \gtrsim a e$, the induced perihelion rate is [84]

$$
\dot{\varpi}_{\mathrm{Yuk}} \approx \frac{\alpha \sqrt{G M a}}{2 \lambda^{2}} \exp \left(-\frac{a}{\lambda}\right)
$$

so that for a pair of planet A and B

$$
\lambda=\frac{a_{\mathrm{B}}-a_{\mathrm{A}}}{\ln \left(\sqrt{\frac{a_{\mathrm{B}}}{a_{\mathrm{A}}}} \Pi_{\mathrm{AB}}\right)} .
$$

The pair A=Earth, B=Mercury allows to obtain [84]

$$
\lambda=0.182 \pm 0.183 \mathrm{AU} .
$$

Solving for $\alpha$ and using $\Delta \dot{\varpi}$ and $a$ for Mars yields [84]

$$
\alpha=\frac{2 \lambda^{2} \Delta \dot{\varpi}}{\sqrt{G M a}} \exp \left(\frac{a}{\lambda}\right)=(0.2 \pm 1) \times 10^{-9},
$$

in which we have used the value for $\lambda$ obtained from Earth and Mercury.

\subsection{Hooke-type theories}

A Hooke-like acceleration is induced, e.g., by a uniform cosmological constant $\Lambda$ in the Schwarzschild-De Sitter spacetime [54]

$$
A_{\Lambda}=\frac{1}{3} \Lambda c^{2} r
$$

The induced perihelion shift is [85]

$$
\dot{\varpi}_{\Lambda}=\frac{1}{2}\left(\frac{\Lambda c^{2}}{n}\right) \sqrt{1-e^{2}}, n=\sqrt{\frac{G M}{a^{3}}} .
$$

Some pairs of planets yield [86]

$$
\begin{gathered}
\Psi_{\text {Mars Mercury }}=7.8 \pm 0.2, \text { ruled out at } 39 \sigma \text { level, } \\
\Psi_{\text {Earth Mercury }}=4.1 \pm 0.2, \text { ruled out at } 20 \sigma \text { level, } \\
\Psi_{\text {Jupiter Mercury }}=51 \pm 12, \text { ruled out at } 4 \sigma \text { level }
\end{gathered}
$$

In the case of the double pulsar, the predicted ratio $\mathscr{R}_{\Lambda}$ between $\dot{\omega}_{\Lambda}$ and $P_{\Lambda}$ is [73]

$$
\mathscr{R}_{\Lambda}=(3.4 \pm 0.3) \times 10^{-8} \mathrm{~s}^{-2} .
$$

Instead, the observed ratio $\mathscr{R}_{\mathrm{obs}}$ between $\Delta \dot{\omega} \equiv \dot{\omega}_{\text {meas }}-\dot{\omega}_{1 \mathrm{PN}}$ and $\Delta P=P_{\mathrm{b}}-P^{\mathrm{Kep}}$ is [73]

$$
\mathscr{R}_{\text {meas }}=(0.3 \pm 4) \times 10^{-11} \mathrm{~s}^{-2} .
$$

They are not compatible at $11 \sigma$ level. 


\subsection{The Pioneer anomaly}

If the Pioneer Anomaly [17, 18] was of gravitational origin, the exotic force causing it should also act on the planets of the Solar System, at least on those moving in the spatial regions in which it manifested itself in its presently known form. A constant and uniform radial acceleration with the same magnitude of that causing the Pioneer Anomaly would induce orbital effects too large [87] to have escaped so far detection, as shown by the $\Delta \dot{\varpi}$ estimated with the EPM2006 ephemerides [67] for Jupiter, Saturn and Uranus [88, 89], and from the residuals of right ascension $\alpha$ and declination $\delta$ computed with the JPL ephemerides [90]. Also some recently proposed velocity-dependent exotic forces $[57,58,60]$ are ruled out by planetary observations $[90,91]$. For other works dealing with the same problem, see Refs. [92, 93, 94, 95].

\subsection{The anomalous perihelion precession of Saturn}

E.V. Pitjeva has recently fitted almost one century of planetary data of all kinds, including also 3 years of radiotechnical data from Cassini, with the EPM2008 ephemerides [16]. She estimated (E.V. Pitjeva, private communication, 2008) a statistically significant non-zero correction

$$
\Delta \dot{\varpi}_{\text {Saturn }}=-0.006 \pm 0.002 \operatorname{arcsec} \mathrm{cy}^{-1},
$$

while the formal error of the fit is $0.0007 \operatorname{arcsec~cy}^{-1}$. However, applying the standard re-scaling by a factor 10 of the optical only observations there is also, in principle, the possibility that the uncertainty can be as large as $0.007 \operatorname{arcsec~cy}^{-1}$ (E.V. Pitjeva, private communication, 2008). Previous results obtained with the EPM2006 ephemerides [67], which did not include Cassini data, yielded

$$
\Delta \dot{\varpi}_{\text {Saturn }}=-0.92 \pm 0.29 \operatorname{arcsec~cy}^{-1} \text { (formal error). }
$$

Is it really a genuine physical effect needing explanation, or is it some artifact of the data reduction procedure? None of the exotic models examined so far is able to accommodate it [15].

\subsection{The general relativistic Lense-Thirring effect}

Until now we have only dealt with putative modifications of the standard Newtonian/Einsteinian laws of gravity. In fact, the estimated corrections $\Delta \dot{\varpi}$ to the usual rates of the perihelia account, by construction, also for a standard general relativistic effect which has not been included in the dynamical force models of the EPM ephemerides used to determine them: it is the gravitomagentic Lense-Thirring effect [96] consisting of secular precessions of the form

$$
\dot{\varpi}_{\mathrm{LT}}=\frac{2 G S(1-3 \cos I)}{c^{2} a^{3}\left(1-e^{2}\right)^{3 / 2}}
$$

where $S$ is the Sun's angular momentum and $I$ is the inclination of the planetary orbital plane to the Sun's equator which is quite small for all the inner planets. The Lense-Thirring precessions for the inner planets are of the order of $10^{-3}-10^{-4} \operatorname{arcsec~cy}^{-1}$, and their direct measurability has been discussed in, e.g., Ref. [97]. It is interesting to check if the unmodelled Lense-Thirring precessions pass the test of the ratio of the perihelia, with

$$
\xi_{\mathrm{AB}}^{\mathrm{LT}} \approx \frac{a_{\mathrm{B}}^{3}\left(1-e_{\mathrm{B}}^{2}\right)^{3 / 2}}{a_{\mathrm{A}}^{3}\left(1-e_{\mathrm{A}}^{2}\right)^{3 / 2}} .
$$


Table 3: Ratios $\xi_{\mathrm{AB}}^{\mathrm{LT}}$ of the predicted Lense-Thirring precessions for the pairs of planets A B.

\begin{tabular}{lll}
\hline A & $\mathrm{B}$ & $\xi_{\mathrm{AB}}^{\mathrm{LT}}$ \\
\hline Mercury & Venus & 7 \\
Venus & Mercury & 0.1 \\
Mercury & Earth & 18 \\
Earth & Mercury & 0.05 \\
Mercury & Mars & 64 \\
Mars & Mercury & 0.01 \\
Venus & Earth & 3 \\
Earth & Venus & 0.4 \\
Venus & Mars & 9 \\
Mars & Venus & 0.1 \\
Earth & Mars & 3.5 \\
Mars & Earth & 0.3 \\
\hline
\end{tabular}

By comparing Table 2 with Table 3, it can be seen that $\Psi_{\mathrm{AB}}$ is compatible with zero for all the pairs A B of inner planets, contrary to all the models of modified gravity examined so far.

\section{Discussion and conclusions}

\subsection{Some technical aspects}

If and when other teams of astronomers will independently estimate their own corrections $\Delta \dot{\varpi}$ with different ephemerides, it will be possible to fruitfully repeat all the tests presented here. In doing them we always used the formal errors in $\Delta \dot{\varpi}$ re-scaled by a factor $\approx 2-5$ for the inner planets and up to 10 times for the outer planets for which mainly optical data have been used. Moreover, in view of the correlations among the estimated $\Delta \dot{\varpi}$, we always linearly propagated their errors, instead of summing them in quadrature, by getting

$$
\delta \Pi_{\mathrm{AB}} \leq\left|\Pi_{\mathrm{AB}}\right|\left(\frac{\delta \Delta \dot{\varpi}_{\mathrm{A}}}{\left|\Delta \dot{\varpi}_{\mathrm{A}}\right|}+\frac{\delta \Delta \dot{\varpi}_{\mathrm{B}}}{\left|\Delta \dot{\varpi}_{\mathrm{B}}\right|}\right)
$$

For example, the correlations between the corrections $\Delta \dot{\Phi}$ of Mercury and the Earth are as large as $20 \%$ (Pitjeva, private communication, 2005). Concerning the uncertainties in $\xi_{\mathrm{AB}}$, in principle, they should have been computed by propagating the errors in the semimajor axes $a$ and the eccentricities $e$ of the planets A and B entering them for each model considered. However, they are quite negligible because the relative (formal) uncertainties in the semimajor axes and eccentricities of the inner planets are all of the order of $10^{-12}$ and $10^{-9}-10^{-11}$, respectively, according to Table 3 of Ref. [67]. Processing more ranging data from Cassini will help in clarifying if the perihelion precession of Saturn can really be considered as a genuine physical effect. 


\subsection{Conclusions}

- All the long-range modified models of gravity examined here are severely challenged either by the ratios of the perihelia of different pairs of solar system's planets or by the double pulsar combined data for the periastron and the orbital period. Both such kinds of ratios cancel out the small multiplicative parameters which directly account for the various exotic models considered, but they still retain a pattern which is characteristic of the model tested through the orbital parameters of the systems used. Only the general relativistic LenseThirring effect, not modelled in the EPM ephemerides and, thus, accounted for, in principle, by the estimated corrections $\Delta \dot{\varpi}$, passes the test of the ratio of the perihelia.

- None of the exotic models examined is able to explain the anomalous perihelion precession of Saturn. The analysis of more Cassini data will help in clarifying if it is really incompatible with zero at some statistically significant level, thus requiring an explanation in terms of some physical phenomena, or if it is some artifact of the data reduction procedure.

\section{Acknowledgments}

I gratefully thank the organizers and the entire staff of this prestigious and high-quality international school for their kind invitation, their exquisite hospitality and the financial support received.

\section{References}

[1] P.S. de Laplace, Traité de Mécanique Céleste, tome IV, livre X, chapitre 7, Courcier, Paris, 1805.

[2] U. Le Verrier, Compt. Rendus de l'Académie des Sciences (Paris) 49, 379 (1859).

[3] A. Hall, Astron. J. 14, 49 (1894).

[4] J. Giné, Chaos, Solitons \& Fractals 38, 1004 (2008), [phys ics / 0510086 ].

[5] A. Einstein, Sitzungsber. Kön. Preuss. Akad. Wiss. (Berlin) 47, 831 (1915).

[6] G. Manev, Compt. Rendus de ľ̌Acadt'emie des Sciences (Paris) 190, 1374 (1930).

[7] E. Fischbach et al., Metrologia 29, 213 (1992).

[8] Y. Fujii, Int. J. Mod. Phys. A 6, 3505 (1991).

[9] H.J. Schmidt, Int. J. Geom. Meth. Mod. Phys 4, 209 (2007), [gr-qc/ 0602017 ].

[10] C. Lämmerzahl, O. Preuss and H. Dittus, Is the physics within the solar system really understood?, in H. Dittus, C. Lämmerzahl and S.G. Turyshev (ed.) Lasers, Clocks and Drag Free Control: Exploration of Relativistic Gravity in Space, Springer, Berlin, 2008, pp. 75-104, [gr-qc/ 0604052 ].

[11] O. Bertolami, Int. J. Mod. Phys. D 16, 2003 (2008), [astro-ph / 0608276 ].

[12] O. Bertolami, J. Paramos, S.G. Turyshev, General Theory of Relativity: Will it survive the next decade? in H. Dittus, C. Lämmerzahl and S.G. Turyshev (ed.) Lasers, Clocks and Drag Free Control: Exploration of Relativistic Gravity in Space, Springer, Berlin, 2008, pp. 27-74, [gr-qc / 0602016 ].

[13] J.D. Anderson, J.K. Campbell and M.M. Nieto, New Astron. 12, 383 (2007), [astro-ph/0608087]. 
[14] J.D. Anderson, J.K. Campbell, J.E. Ekelund, J. Ellis and J.F. Jordan, Phys. Rev. Lett. 100, 091102 (2008).

[15] L. Iorio, Astron. J. 137, 3615 (2009), [arXiv : 0811.0756$].$

[16] E.V. Pitjeva, Ephemerides EPM2008: the updated models, constants, data paper presented at Journées "Systèmes de référence spatio-temporels" and X Lohrmann-Kolloquium 22-24 September 2008, Dresden, Germany.

[17] J.D. Anderson, P.A. Laing, E.L. Lau, A.S. Liu, M.M. Nieto and S.G. Turyshev, Phys. Rev. Lett. 81, 2858 (1998), [gr-qc/9808081].

[18] J.D. Anderson, P.A. Laing, E.L. Lau, A.S. Liu, M.M. Nieto and S.G. Turyshev, Phys. Rev. D 65, 082004 (2002), [gr-qc/ 0104064 ].

[19] F. Zwicky, Helvetica Physica Acta 6, 110 (1933).

[20] F.D. Kahn and L. Woltjer, 1959, Astrophys. J. 130, 705 (1959).

[21] A. Bosma, Astron. J. 86, 1791 (1981).

[22] V. Rubin, W. Ford, N. Thonnard and D. Burstein, 1982, Astroph. J. 261, 439 (1982).

[23] V. Rubin, Science 220, 1339 (1983).

[24] M. Persic, P. Salucci and F. Stel, Mon. Not. Roy. Astron. Soc. 281, 27 (1996), [astro-ph/9506004].

[25] A.G. Riess et al., Astron. J. 116, 1009 (1998),[astro-ph/9805201].

[26] S. Perlmutter et al., Astrophys. J. 517, 565 (1999), [astro-ph/9812133].

[27] A.S. Friedman and J.S. Bloom, Astrophys. J. 627, 1 (2005), [ast ro-ph/ 0408413 ].

[28] W.J. Percival et al., Mon. Not. Roy. Astron. Soc. 381, 1053 (2007), [arXiv : 0705.3323 ].

[29] E. Komatsu et al., Astrophys. J. Suppl. 180, 330 (2009), [arXiv: 0803.0547$].$

[30] G. Efstathiou et al., Mon. Not. Roy. Astron. Soc. 330, L29 (2002), [astro-ph/0109152]

[31] L. Fu et al., Astron. Astrophys. 479, 1 (2008), [arXiv: 0712 . 0884].

[32] L. Perivolaropoulos, Six Puzzles for LCDM Cosmology, in P.M. Lavrov (ed.) The problems of modern cosmology. A volume in honour of Professor S.D. Odintsov on the occasion of his 50th birthday, Tomsk State Pedagogical University Press, Tomsk, 2009, pp. 245-254, [arXiv: 0811 . 4684].

[33] G. Dvali, G. Gabadadze and M. Porrati, Phys. Lett. B 485, 208 (2000), [hep-th/ 0005016 ].

[34] J.W. Moffat, J. Cosmol. Astropart. Phys. 2006, 004 (2006), [gr-qc/ 0506021$].$

[35] J.W. Moffat, Int. J. Mod. Phys. D 16, 2075 (2008), [gr-qc/ 0608074 ].

[36] M. Milgrom, Astrophys. J. 270, 365 (1983).

[37] M. Milgrom, Astrophys. J. 270, 371 (1983).

[38] M. Milgrom, Astrophys. J. 270, 384 (1983).

[39] K. Begeman, A. Broeils and R. Sanders, Mon. Not. Roy. Astron. Soc. 249, 523 (1991).

[40] T.P. Sotiriou and V. Faraoni, [arXiv:0805.1726].

[41] S. Capozziello and M. Francaviglia, Gen. Relativ. Gravit. 40, 357 (2008), [arXiv : 0706.1146 ]. 
[42] I. Navarro and K. van Acoleyen, Phys. Lett. B 622, 1 (2005), [gr-qc/ 0506096 ].

[43] I. Navarro and K. van Acoleyen, J. Cosmol. Astropart. Phys. 2006, 008 (2006), [gr-qc/ 0511045 ].

[44] I. Navarro and K. van Acoleyen, J. Cosmol. Astropart. Phys. 2006, 006 (2006), [gr-qc/ 0512109 ].

[45] D.E. Krause and E. Fischbach, Searching for extra-dimensions and new string-inspired forces in the Casimir regime, in C. Lämmerzahl, C.W.F. Everitt and F.W. Hehl (ed.) Gyros, Clocks, Interferometers. . . : Testing Relativistic Gravity in Space Springer, Berlin, 2001, pp. 292-309, [hep-ph/9912276].

[46] E.G. Adelberger, B.R. Heckel and A.E. Nelson, Ann. Rev. Nucl. Part. Sci. 53, 77 (2003), [hep-ph/0307284].

[47] M. White and C.S. Kochanek, Astrophys. J. 560, 539 (2001), [astro-ph/0105227].

[48] L. Amendola and C. Quercellini, Phys. Rev. Lett. 92, 181102 (2004), [a stro-ph/ 0403019 ].

[49] C. Sealfon, L. Verde and R. Jimenez, Phys. Rev. D 71, 083004 (2005), [ast ro-ph/ 0404111 ].

[50] S. Reynaud and M.-T. Jaekel, Int. J. Mod. Phys. A 20, 2294 (2005), [gr-qc/ 0501038 ].

[51] A. Shirata, T. Shiromizu, N. Yoshida and Y. Suto, Phys. Rev. D 71, 064030 (2005), [astro-ph/0501366].

[52] M. Sereno and J.A. Peacock, Mon. Not. Roy. Astron. Soc. 371, 719 (2006), [a st ro-ph/ 0605498 ].

[53] Z. Stuchlík and S. Hledík, Phys. Rev. D 60, 044006 (1999).

[54] W. Rindler, Relativity: Special, General, and Cosmological Oxford University Press, Oxford, 2001.

[55] M.-T. Jaekel and S. Reynaud, Class. Quantum Grav. 23, 7561 (2006), [gr-qc/0610155].

[56] J.R. Brownstein and J.W. Moffat, Class. Quantum Grav. 23, 3427 (2006), [gr-qc/ 0511026 ].

[57] M.-T. Jaekel and S. Reynaud, Mod. Phys. Lett. A 20, 1047 (2005), [gr-qc/ 0410148 ].

[58] M.-T. Jaekel and S. Reynaud, Class. Quantum Grav. 22, 2135 (2005), [gr-qc/ 0502007 ].

[59] M.-T. Jaekel and S. Reynaud, Class. Quantum Grav. 23, 777 (2006), [gr-qc/0510068].

[60] E.M. Standish, Planetary and Lunar Ephemerides: testing alternate gravitational theories, in A. Macias, C. Lämmerzahl and A. Camacho (ed.) AIP Conference Proceedings Volume 977. RECENT DEVELOPMENTS IN GRAVITATION AND COSMOLOGY: 3rd Mexican Meeting on Mathematical and Experimental Physics, American Institute of Physics, 2008, pp. 254-263.

[61] T. Jacobson and D. Mattingly, Phys. Rev. D 64, 024028 (2001), [gr-qc/ 0007031 ].

[62] J.D. Bekenstein, Phys. Rev. D 70, 083509 (2004), [astro-ph/ 0403694 ].

[63] R. Maartens, Living Rev. Rel. 7, 7 (2004), [gr-qc/0312059].

[64] M. Burgay, N. D’ Amico, A. Possenti, R.N. Manchester, A.G. Lyne, B.C. Joshi, M.A. McLaughlin, M. Kramer, J.M. Sarkissian, F. Camilo, V. Kalogera, C. Kim and D.R. Lorimer, Nature 426, 531 (2003), [astro-ph/0312071].

[65] M. Kramer, I.H. Stairs, R.N. Manchester, M.A. McLaughlin, A.G. Lyne, R.D. Ferdman, M. Burgay, D.R. Lorimer, A. Possenti, N. D’Amico, J.M. Sarkissian, G.B. Hobbs, J.E. Reynolds, P.C.C. Freire and F. Camilo, Science 314, 97 (2006), [astro-ph/ 0609417 ].

[66] E.V. Pitjeva, Astron. Lett. 31, (2005). 
[67] E.V. Pitjeva, Use of optical and radio astrometric observations of planets, satellites and spacecraft for ephemeris astronomy, in W.J. Jin, I. Platais and M.A.C. Perryman (ed.) A Giant Step: from Milli- to Micro-arcsecond Astrometry Proceedings IAU Symposium No. 248, 2007, Cambridge University Press, Cambridge, 2008, pp. 20-22.

[68] E.V. Pitjeva, Sol. Sys. Res. 39, 176 (2005).

[69] A. Lue and G. Starkman, Phys. Rev. D 67, 064002 (2003), [astro-ph/0212083].

[70] G. Dvali, A. Gruzinov and M. Zaldarriaga, Phys. Rev. D 68, 024012 (2003), [hep-ph/ 0212069 ].

[71] L. Iorio, Class. Quantum Grav. 22, 5271 (2005), [gr-qc/ 0504053 ].

[72] L. Iorio, Adv. High En. Phys. 2007, 90731 (2007), [arXiv: 0710 . 0022].

[73] L. Iorio, New Astron. 14, 196 (2009), [arXiv: 0808 . 0256].

[74] J.W. Moffat and V.T. Toth, Astrophys. J. 680, 1158 (2008), [arXiv: 0708 . 1935].

[75] J.W. Moffat and V.T. Toth, Class. Quantum Grav. 26, 085002 (2009), [arXiv: 0712 . 1796].

[76] L. Iorio, Schol. Res. Exchange 2008, 238385 (2008), [arXiv : 0809 . 3563].

[77] J. Bekenstein and M. Milgrom, Astrophys. J. 286, 7 (1984).

[78] B. Famaey and J. Binney, Mon. Not. Roy. Astron. Soc. 363, 603 (2005), [ast ro-ph / 0506723 ].

[79] C. Talmadge, J.-P. Berthias, R.W. Hellings and E.M. Standish, Phys. Rev. Lett. 61, 1159 (1988).

[80] M. Sereno and Ph. Jetzer, Mon. Not. Roy. Astron. Soc. 371, 626 (2006), [a st ro-ph/ 0606197 ].

[81] L. Iorio, J. Gravit. Phys. 2, 26 (2008), [arXiv: 0711.2791$].$

[82] S. Capozziello, V.F. Cardone and A. Troisi, Mon. Not. Roy. Astron. Soc. 375, 1423 (2007), [astro-ph/0603522].

[83] L. Iorio and M.L. Ruggiero, Schol. Res. Exchange 2008, 968393 (2008), [arXiv : 0711 . 0256 ].

[84] L. Iorio, J. High En. Phys. 2007, 041 (2007), [arXiv: 0708 . 1080].

[85] A.W. Kerr, J.C. Hauck and B. Mashhoon, Class. Quantum Grav. 20, 2727 (2003), [gr-qc/0301057].

[86] L. Iorio, Adv. Astron. 2008, 268647 (2008), [arXiv: 0710.2610$].$

[87] L. Iorio and G. Giudice, New Astron. 11, 600 (2006), [gr-qc/ 0601055$].$

[88] L. Iorio, THE LENSE-THIRRING EFFECT AND THE PIONEER ANOMALY: SOLAR SYSTEM TESTS, in H. Kleinert, R.T. Jantzen and R.J. Ruffini (ed.) THE ELEVENTH MARCEL GROSSMANN MEETING On Recent Developments in Theoretical and Experimental General Relativity, Gravitation and Relativistic Field Theories. Proceedings of the MG11 Meeting on General Relativity Berlin, Germany 23-29 July 2006, Singapore, World Scientific, 2008, pp. 2558-2560, [gr-qc/ 0608105 ].

[89] L. Iorio, J. Gravit. Phys. 1, 1 (2007), [arXiv:0712.1273].

[90] L. Iorio, Found. Phys. 37, 897 (2007), [gr-qc/ 0610050 ].

[91] L. Iorio, Int. J. Mod. Phys. D at press, [arXiv:0806.3011].

[92] G. Page, D.S. Dixon, J.F. Wallin, Astrophys. J. 642, 606 (2006), [astro-ph / 0504367 ].

[93] K. Tangen, Phys. Rev. D 76, 042005 (2007), [gr-qc/ 0602089 ].

[94] J.F. Wallin, D.S. Dixon and G.L. Page, Astrophys. J. 666, 1296 (2007), [arXiv: 0705 . 3408 ]. 
[95] G. Page, J.F. Wallin, D.S. Dixon, Astrophys. J. 697, 1226 (2009), [arXiv: 0905 . 0030 ].

[96] J. Lense and H. Thirring, Phys. Z. 19, 156 (1918).

[97] L. Iorio, Proc. of Science ISFTG, 017 (2009), [arXiv : 0905 . 0300]. 